\title{
Response to Cabergoline treatment in Invasive Prolactinoma with hemorrhage- A case report
}

Vivek Pant', Suman Baral'2, Binod Aryal', Arjun Tumbapo'

'Department of Biochemistry, Institute of Medicine, Maharajgunj, Kathmandu, ${ }^{2}$ Endocrinology Unit, Institute of Medicine, Maharajgunj, Kathmandu

\begin{abstract}
Patients with invasive prolactinoma present with constellation of symptoms including headache, blurred vision, lethargy, menstrual irregularity and sexual dysfunction. Cabergoline, a potent dopamine agonist, is a known medication prescribed for the treatment of prolactinoma. Here, we report a case of invasive macroprolactinoma with hemorrhage in a 18 years female with dramatic response to cabergoline treatment clinically, biochemically, and radiologically.
\end{abstract}

Keywords : cabergoline; prolacti ; prolactinoma

\section{INTRODUCTION}

Pituitary adenomas are benign tumors of the pituitary gland, with lactotroph cell adenoma (prolactinoma) being the commonest, accounting for $44 \%$ of known cases.1 Microprolactinomas are less than 1 $\mathrm{cm}$ in diameter whereas macroadenomas measures more than $1 \mathrm{~cm} .1$ Invasive giant macroadenoma are defined as tumor size above $4 \mathrm{~cm}$ and serum prolactin more than $1000 \mathrm{ng} / \mathrm{mL}$ with clinical symptoms of hyperprolactinemia or mass effect. Prolactinomas come to attention because of the effect of the elevated prolactin on the reproductive system and/or due to the compression effect of the tumor.

Dopamine agonists reduce the size of prolactinomas by inducing a reduction in cell volume via inhibition of secretory mechanism as well as causing perivascular fibrosis and cell necrosis. 2 It has been demonstrated that cabergoline is safe and effective for treatment of invasive giant prolactinomas, even when administered in relatively high doses for longer duration.

In this case, we describe the case of a 18 years Nepalese female diagnosed with macroprolactinoma with hemorrhage and its dramatic response to dopamine agonist (cabergoline).

\section{CASE REPORT}

Eighteen years old female from kathmandu presented to the endocrinology OPD of Tribhuvan university teaching hospital on 26th January 2016 with complains of headache and decreased bilateral vision for 3 months. She also had generalized fatigability, lethargy and menstrual irregularities for 2 years. She also reported history of galactorrhea on and off. There is no history of hearing loss, abnormal movements of body or altered level of consciousness. Family history was unremarkable.

On physical examination, patient was conscious. Vital signs were stable. Thyroid examination was normal. Perimetry examination showed bilateral superior quadrantanopia with full range of extra ocular muscle movements. Systemic examination was unremarkable. Laboratory investigations showed hyperprolactinemia. Initially, serum prolactin was $>320 \mathrm{ng} / \mathrm{mL}$. Then after 200 times dilution serum prolactin value was $49,680 \mathrm{ng} / \mathrm{mL}$ (normal value $=3-18.6 \mathrm{ng} / \mathrm{mL}$ ). Other pituitary work-up was normal. Serum cortisol at 8 a.m and overnight dexamethasone suppression test were within normal range. There was no finding suggestive of hypogonadism, hypothyroidism, and adrenal insufficiency. Plain Magnetic resonance imaging (MRI) of head was done which revealed a mass of 2.2 
* $1.5 \mathrm{~cm}$ within the pituitary fossa. Mass was extending superiorly and compressing optic chiasma. High signal intensity was noted due to hemorrhage (Fig. 1). The patient was thus diagnosed with pituitary macroadenoma with hemorrhage. She was then started on cabergoline $0.25 \mathrm{mg}$ twice weekly and was advised to follow up after one month with laboratory reports of serum prolactin.

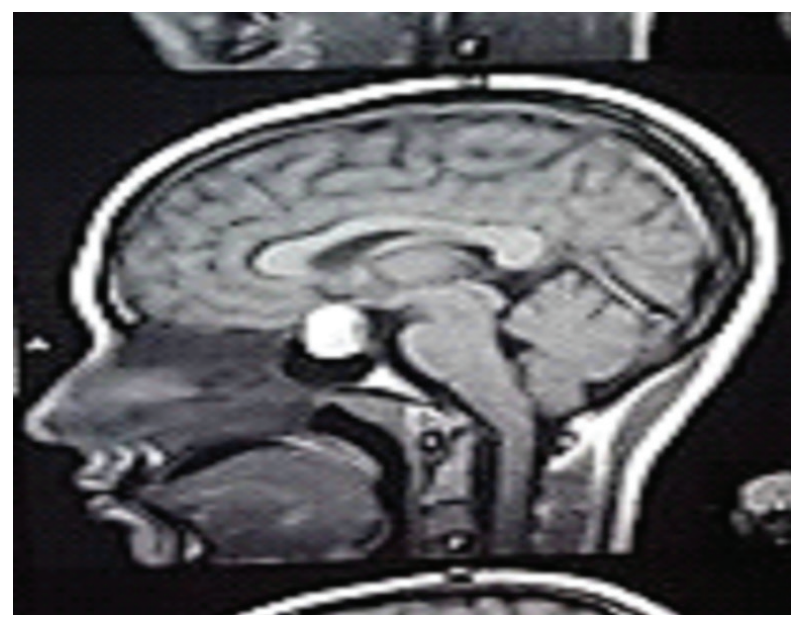

Figure 1. Pituitary macroadenoma with hemorrhage

After one month on 4th March 2016, serum prolactin levels dropped to $1152.0 \mathrm{ng} / \mathrm{mL}$ (3$18.6 \mathrm{ng} / \mathrm{mL}$ ), with subsequent improvements in vision and headache. Perimetry test showed normal results. The patient was then prescribed with higher dose of cabergoline $1.25 \mathrm{mg}$ twice a week and was advised to follw up after 3 months. Gradually her serum prolactin dropped down to high normal range after one and half year of treatment. She regained her normal menstrual cycle. On 31st July 2017. her serum prolactin was $19.0 \mathrm{ng} / \mathrm{mL}$ and her repeated MRI of brain with pituitary showed complete disappearence of pituitary lesion as compared to earlier imaging (Fig. 2).

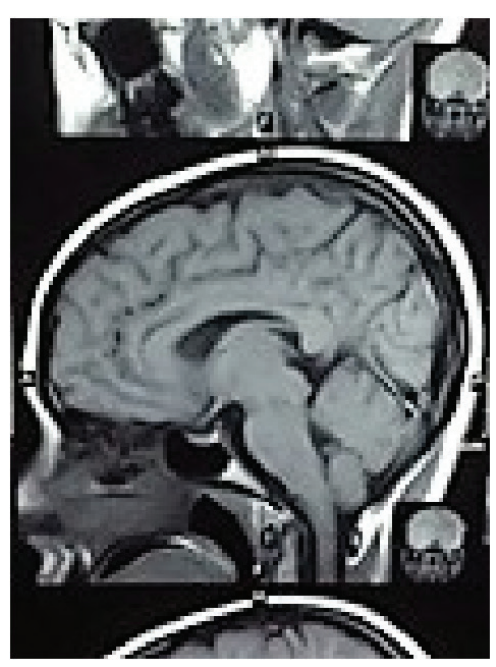

Fig 2. Normal Pituitury MRI after one and half years of treatment

\section{DISCUSSION}

Treatment of invasive giant prolactinoma with locoregional spread and visual field compromise using cabergoline has been observed to provide excellent outcomes. Our case illustrates the efficacy of cabergoline in treating invasive macroprolactinoma with hemorrhage and reversing patient's symptoms.

In female, prolactinoma is usually diagnosed earlier than men because of presence of menstrual distubances due to hyperprolactinemia. Macroadenoma compresses pituitary stalk causing loss of dopamine inhibitory tone to the lactotroph and subsequent hyperprolactinemia. Our patient had initially presented with irregular menses in various hospitals but her symptoms were overlooked. Finally she presented to us with visual disturbances and headache.

In our case, serum prolactin was more than $320 \mathrm{ng} / \mathrm{mL}$ during her first visit to us. After dilution of the sample, serum prolactin value was $49,680 \mathrm{ng} / \mathrm{mL}$. The Endocrine Society's 
Clinical Guideline "Diagnosis and Treatment of Hyperprolactinemia" in 2011 recommends that in case of discrepancy between a very large pituitary tumor and a mildly elevated prolactin level, serial dilution of serum samples need to be performed to eliminate an artifact leading to a falsely low prolactin value the so called "hook effect" or high dose hook (HDH) effect.3

Significant improvements were observed in our case after an initial dose of $0.25 \mathrm{mg}$ of cabergoline twice weekly. Also, patient showed dramatic improvement in visual field symptoms within the first month of therapy. Her menstrual cycle was normal after one and half years of treatment. During this period her serum prolactin was near normal. Various cases has been reported where treatment with cabergoline for 18 months markedly reduced tumor size and visual field improvement.4,5 Transsphenoidal surgery is indicated only for patients who are resistant to dopamine agonist treatment.6 Also, the cure rate for macroprolactinoma and microprolactinoma treated with surgery is poor which is $30 \%$ and $80 \%$ respectively and there is a risk of hypopituitarism and recurrence.6 Occasionally, surgery may be required for patients with CSF leak. Cabergoline is more effective than other dopamine agonist like bromocriptine in normalization of serum prolactin in pituitary microadenoma.

This case reinforces the effectiveness of cabergoline treatment and emphasizes the potential reversibility of pituitary function in patients harboring invasive and aggressive prolactin-secreting pituitary tumors with hemorrhage. This case also supports medical treatment over surgery for prolactinomas and demonstrates that cabergoline is a safe and effective treatment and should be recommended as a first line therapy.

\section{CONCLUSIONS}

Cabergoline has been successfully used in treatment of invasive giant prolactinoma. This case supports medical treatment over surgery for prolactinomas with hemorrhage.

\section{CONFLICT OF INTEREST}

Authors declare that there is no conflict of interest in the publication of this case report.

\section{REFERENCES}

1. Siddiqui A, Chew N, Miszkiel K. Unusual orbital invasion by a giant prolactinoma. The British journal of radiology. 2008 Nov;81 (971): e259-62.

2. Bevan JS, Webster J, Burke CW, Scanlon MF. Dopamine agonists and pituitary tumor shrinkage. Endocrine reviews. 1992 May 1;13(2):220-40.

3. Melmed S, Casanueva FF, Hoffman AR, Kleinberg DL, Montori VM, Schlechte JA, Wass JA. Diagnosis and treatment of hyperprolactinemia: An Endocrine Society clinical practice guideline. The Journal of Clinical Endocrinology \& Metabolism. 2011 Feb 1:96(2):273-88.

4. Dutta D, Ghosh S, Mukhopadhyay S, Chowdhury S. Rapid reduction with cystic transformation of invasive giant prolactinoma following short term low dose cabergoline. Indian journal of endocrinology and metabolism. 2012 Nov; 16(6):1048.

5. Alsubaie S, Almalki MH. Cabergoline treatment in invasive giant prolactinoma. Clinical medicine insights. Case reports. 2014; 7:49.

6. Menucci M, Quiñones-Hinojosa A, Burger $P$, Salvatori R. Effect of dopaminergic drug treatment on surgical findings in prolactinomas. Pituitary. 2011 Mar 1;14(1):68-74. 